\title{
Scalar Field Cosmology in Three-Dimensions
}

\author{
G. Oliveira-Neto* \\ Departamento de Física, Instituto de Ciências Exatas, \\ Universidade Federal de Juiz de Fora, \\ 36036-330, Juiz de Fora, MG, Brazil \\ Received on 17 October, 2000
}

\begin{abstract}
We study an analytical solution to the Einstein's equations in 2+1-dimensions. The space-time is dynamical and has a line symmetry. The matter content is a minimally coupled, massless, scalar field. Depending on the value of certain parameters, this solution represents three distinct spacetimes. The first one is flat space-time. Then, we have a big bang model with a negative curvature scalar and a real scalar field. The last case is a big bang model with event horizons where the curvature scalar vanishes and the scalar field changes from real to purely imaginary.
\end{abstract}

\section{Introduction}

Many recent works have focused attention on the issue of general relativity in $2+1$-dimensions [1]. Most of them deal with two important issues: black holes and cosmology.

The main motivation for the most recent works in black hole physics came from the important discovery of the BTZ black hole [2]. They investigate different classical and quantum properties of the BTZ and other black hole solutions [3], [4].

On the other hand, the simplicity of the theory in three-dimensions, when compared with its version in four-dimensions, is the main motivation for the works in cosmology. Most of them deal with quantum aspects of the theory, in particular the attempt to derive a wave-function for the Universe [4], [5], [6].

In the present work we would like to study an analytical solution to the Einstein's equations in $2+1-$ dimensions. The space-time is dynamical and has a line symmetry. The matter content is represented by a minimally coupled, massless, scalar field. We shall see that this solution gives rise, depending on the value of certain parameters, to three different space-times. The first one is flat space-time. Then, we have a big bang model with a negative curvature scalar and a real scalar field. The last case is a big bang model with event horizons where the curvature scalar vanishes and the scalar field changes from real to purely imaginary.

In Sec. II, we start by writing down the appropriate Einstein's equations. A solution $(S)$ of this set of equations has already been found, in different circum- stances Ref. [7]. We shall use $S$ suitably adapted to our problem.

$S$ is composed of few functions of the relevant coordinates and free parameters $\left(p_{i}\right)$. In Sec. III, we impose conditions upon $S$ such that it may be interpreted as a physically acceptable solution. These conditions restrict the domains of the $p_{i}$ 's, and divide $S$ in three distinct types of space-times, depending on the values of the $p_{i}$ 's. The space-times are the ones mentioned above and we study them in great details.

Finally, in Sec. IV we summarize the main points and results of the paper.

\section{Einstein-scalar equations}

We shall start by writing down the ansatz for the 2 + 1-dimensional, dynamical, line symmetric, spacetime metric as,

$$
d s^{2}=-2 e^{(N(u, v)+U(u, v) / 2)} d u d v+e^{-2 U(u, v)} d y^{2} .
$$

where $N(u, v)$ and $U(u, v)$ are two arbitrary functions to be determined by the field equations, $(u, v)$ is a pair of null coordinates varying in the range $(-\infty, \infty)$, and $y$ is a coordinate taking values over a real line: $(-\infty, \infty)$.

The scalar field $\varphi$ will be a function only of the two null coordinates and the expression for its stress-energy tensor $T_{\alpha \beta}$ is given by [8],

$$
T_{\alpha \beta}=\varphi,_{\alpha} \varphi,_{\beta}-\frac{1}{2} g_{\alpha \beta} \varphi,_{\lambda} \varphi^{, \lambda} .
$$

where, denotes partial differentiation.

\footnotetext{
*Email: gilneto@fisica.ufjf.br
} 
Now, combining Eqs. (1) and (2) we may obtain the Einstein's equations, which in the units of Ref. [8] take the following form,

$$
\begin{gathered}
2 U,_{u u}-\left(U,_{u}\right)^{2}+2 N,_{u} U,_{u}=2(\varphi, u)^{2}, \\
2 U_{, v v}-\left(U,_{v}\right)^{2}+2 N,_{v} U,_{v}=2(\varphi, v)^{2}, \\
2 N,_{u v}+U,_{u v}=2 \varphi,_{u} \varphi,_{v}, \\
\left(e^{U}\right)_{, u v}=0 .
\end{gathered}
$$

The equation of motion for the scalar field, in these coordinates, is

$$
2 \varphi,{ }_{u v}-U,{ }_{u} \varphi,{ }_{v}-U,_{v} \varphi,,_{u}=0,
$$

Comparing the above set of non-linear, secondorder, coupled, partial, differential equations (3)-(7) with the one derived in Ref. [7], called type $A$, we notice that they are identical, although the situation treated here is different from the one treated there. Therefore, we shall take as the solution of the system above Eqs. (3)-(7), the type $A$ space-times, obtained in Ref. [7]. They will be introduced and studied in great details in the next section.

\section{Solutions}

From our discussion in the previous section, we suitably adapt the type $A$ space-times of Ref. [7] to the present situation, and write the following solution to the system Eqs. (3)-(7),

$$
\begin{gathered}
\exp (-U(u, v)) \equiv t^{2}(u, v)=\left(1-u^{2 \eta}-v^{2 \mu}\right)^{2} \\
\exp (-N(u, v)) \equiv W(u, v)=\quad t^{2 \delta}\left[\frac{u^{\eta} v^{\mu}-\left(1-v^{2 \mu}\right)^{1 / 2}\left(1-u^{2 \eta}\right)^{1 / 2}}{u^{\eta} v^{\mu}+\left(1-v^{2 \mu}\right)^{1 / 2}\left(1-u^{2 \eta}\right)^{1 / 2}}\right]^{2 \gamma}\left[\frac{u^{\mu}-\left(1-v^{2 \mu}\right)^{1 / 2}}{u^{\eta}+\left(1-v^{2 \mu}\right)^{1 / 2}}\right]^{4 a \delta_{+}} \\
\times\left[\frac{v^{\mu}-\left(1-u^{2 \eta}\right)^{1 / 2}}{v^{\mu}+\left(1-u^{2 \eta}\right)^{1 / 2}}\right]^{4 a \delta_{-}}\left(1-v^{2 \mu}\right)^{-\delta_{+}^{2}}\left(1-u^{2 \eta}\right)^{-\delta_{-}^{2}}, \\
\varphi(u, v)=\quad 2 a \ln t^{2}+\rho_{+} \ln \left[\frac{1-u^{\eta}\left(1-v^{2 \mu}\right)^{1 / 2}-v^{\mu}\left(1-u^{2 \eta}\right)^{1 / 2}}{1+u^{\eta}\left(1-v^{2 \mu}\right)^{1 / 2}+v^{\mu}\left(1-u^{2 \eta}\right)^{1 / 2}}\right] \\
+\rho_{-} \ln \left[\frac{1-u^{\eta}\left(1-v^{2 \mu}\right)^{1 / 2}+v^{\mu}\left(1-u^{2 \eta}\right)^{1 / 2}}{1+u^{\eta}\left(1-v^{2 \mu}\right)^{1 / 2}-v^{\mu}\left(1-u^{2 \eta}\right)^{1 / 2}}\right]
\end{gathered}
$$

where $\mu$ and $\eta \in \Re$ and they are greater than or equal to $1 / 2, \delta=2 a^{2}+(3-1 / \eta-1 / \mu) / 4, \gamma^{2}=$ $(1-1 / 2 \eta)(1-1 / 2 \mu), \delta_{+}^{2}=1-1 / 2 \eta, \delta_{-}^{2}=1-1 / 2 \mu$, $\rho_{ \pm}=(1 / 2)\left(\delta_{+} \pm \delta_{-}\right)$, and $a$ is a constant, real, number.

In terms of the new quantities $t(u, v)$ Eq. (8), and $W(u, v)$ Eq. (9), the line element Eq. (1) becomes,

$$
d s^{2}=-2 W(u, v) t^{1 / 2}(u, v) d u d v+t^{2}(u, v) d y^{2},
$$

One may notice from Eqs. (8)-(10), that for different values of $\eta, \mu$ and $a$, one has different space-times. It is also important to notice that the choice of the letter $t$ in Eq. (8), was not casual. We shall be able to interpret it as a time coordinate.

Observing Eq. (11), we notice that these spacetimes have a singularity at $t=0$. It is a physical singu- larity as can be seen directly from the curvature scalar $R$, and also from Ref. [7].

In order to show this result we start writing down the Ricci tensor that, in the present case, has the following expression [9],

$$
R_{\alpha \beta}=\varphi, \alpha \varphi, \beta \text {. }
$$

From it, we may compute $R$ straightforwardly with the aid of Eqs. (8)-(11), finding,

$$
R=\frac{-8 \mu \eta v^{2 \mu-1} u^{2 \eta-1}\left(4 a+\delta_{+}+\delta_{-}\right)^{2}}{W t^{5 / 2}} .
$$

Finally, taking the limit $t \rightarrow 0$ in $R$ Eq. (13), we find that this quantity diverges at $t=0$. There is no 
other physical singularity for these space-times because $R$ is well defined outside $t=0$. We can also learn that taking the limit $t \rightarrow \infty$ of $R$ Eq. (13), this quantity goes to zero.

The space-times above will only be physically acceptable if $t$ Eq. (8) is a real, positive function and $W$ Eq. (9) is a real function. Therefore, only few distinct sets of values of $\mu, \eta$ and $a$ will be allowed. Each of them giving rise to a different type of space-time. It is important to note that since $t$ and $W$ are functions of $(u, v)$, the range of these coordinates shall also be restricted.

Here we shall loose the condition that the scalar field $\varphi$ Eq. (10), be real. We shall permit it to be purely imaginary in some space-time regions, which means that in those regions $\varphi$ will be an example of the so-called 'exotic matter' [10].

\section{III.1 Space-times}

We start now the determination of the allowed values of $\mu, \eta$ and $a$ by imposing that $t$ be a real, positive, function. In order to simplify our study we shall demand that $2 \mu$ and $2 \eta$ be integers, greater than or equal to 1 .

Observing the definition of $t$ Eq. (8), we notice that if we permit $2 \mu$ or $2 \eta$ to be even, for positive $t$, we would have very limited domains for the coordinates $u$ and $v$, respectively. Since we would like to have the biggest possible domains for these coordinates, we shall restrict our attention to odd, integer, values of $2 \mu$ and $2 \eta$. The positiveness of $t$ will also select the physically accessible space-time volume.

From the expression of W Eq. (9), we learn that the three distinct functions of $u$ and $v$, inside brackets, respectively with exponents $2 \gamma, 4 a \delta_{+}$and $4 a \delta_{-}$, may be negative even for positive $t$. Since, in order to interpret $t$ as a time coordinate, $W$ has to be positive (see Eq. (14) below), we shall eliminate these terms. The best way to accomplish this is by setting theirs exponents to zero.

With the aid of the formulae for $\gamma, \delta_{+}$and $\delta_{-}$, given just below Eq. (10), we understand that there are three distinct manners to set the above exponents to zero: (a) by choosing $a=0$ and $2 \mu=2 \eta=1$, (b) by choosing $2 \mu=2 \eta=1$, and finally (c) by choosing $a=0$ and either $2 \mu$ or $2 \eta$ equal to 1 . As we shall see below, these possibilities will produce the distinct sets of space-times associated to our solution.

Now, we are in the position to identify $t$ as a time coordinate. For positive $W$ and $t$, we may compute the norm of the quadri-vector normal to surfaces of constant $t$. Which gives,

$$
-8 \eta \mu u^{2 \eta-1} v^{2 \mu-1} \frac{1}{W t^{1 / 2}},
$$

which is always negative for $2 \eta$ and $2 \mu$, odd, integer, numbers.

Gathering together all the conditions obtained above, we may group the solutions satisfying these conditions in three distinct sets.

\section{III.1.1 Flat space-time.}

The first is empty, flat space-time, obtained for $2 \mu=2 \eta=1$ and $a=0$. It has the following line element, from Eqs. (8), (9) and (11),

$$
d s^{2}=-2 d u d v+t^{2} d y^{2},
$$

where, $t=1-u-v$.

For positive $t$, the coordinates $u$ and $v$ will vary in the range $(-\infty, \infty)$, but under the condition,

$$
u+v<1 .
$$

The surface $t=0$ in this case is not a physical singularity, it is just a coordinate singularity.

\section{III.1.2 Big bang cosmology without horizons.}

The solutions belonging to this set have $2 \mu=2 \eta=1$ and $a \neq 0$. Introducing these values in Eqs. (8), (9) and (11), we may write the following line element,

$$
d s^{2}=-2 t^{4 a^{2}} d u d v+t^{2} d y^{2},
$$

where $t=1-u-v, u, v \in(-\infty, \infty)$ and satisfy Eq. (16).

The scalar field Eq. (10) is now,

$$
\varphi=2 a \ln t^{2} .
$$

In the present case, we may see from Eq. (18) that the scalar field is always real, therefore the stress-energy Eq. (2) is always positive.

The scalar of curvature Eq. (13), is written

$$
R=-\frac{32 a^{2}}{t^{\left(4 a^{2}+2\right)}} \text {. }
$$

From this expression is easy to see that $t=0$ is still a physical singularity for these space-times. The scalar field Eq. (18), is also singular there. Therefore, we may interpret this singularity as a big bang, for this space-time.

The dynamical nature of this space-time may be better appreciated if we re-write the line element Eq. (17) in terms of $t$ and $x=u-v$,

$$
d s^{2}=\frac{t^{4 a^{2}}}{2}\left(-d t^{2}+d x^{2}\right)+t^{2} d y^{2},
$$


where $-\infty<x<\infty$.

\section{III.1.3 Big bang cosmology with event horizons}

The last set of space-times is determined when we set $a=0$ and either $2 \mu$ or $2 \eta$ equal to 1 . As a matter of definition, and without loosing the generality, let us choose $2 \eta=1$ and $2 \mu=2 n+1$, where $n$ is a positive integer.

With the aid of Eqs. (8), (9) and (11), the line element of these space-times is,

$$
d s^{2}=-2\left(\frac{t}{1-u}\right)^{\left(\frac{2 n}{2 n+1}\right)} d u d v+t^{2} d y^{2},
$$

where $t=1-u-v^{2 n+1}, u, v \in(-\infty, \infty)$ and satisfy the condition $u+v^{2 n+1}<1$.

Observing Eq. (21), we identify besides the singularity at $t=0$, another one at $u=1$. The second singularity is not a physical one as can be seen directly from $R \mathrm{Eq}$. (13), which for the present situation is,

$$
R=\frac{-4 n v^{2 n}(1-u)^{\left(\frac{2 n}{2 n+1}\right)}}{t^{\left(\frac{6 n+2}{2 n+1}\right)}} .
$$

Indeed, $u=1$ is an event horizon as we shall demonstrate below.

Since $u=1$ is just a coordinate singularity, there are new coordinates which let Eq. (21) regular at this event. On the other hand, in order to better understand the physical effect of the horizon and the dynamical nature of the space-time, it is more convenient to re-write the line element Eq. (21) in terms of, $t$ and $x=-1+u-v^{2 n+1}$. Which gives,

$$
d s^{2}=\frac{1}{4 n+2}\left(\frac{4 t}{x^{2}-t^{2}}\right)^{\left(\frac{2 n}{2 n+1}\right)}\left(-d t^{2}+d x^{2}\right)+t^{2} d y^{2} .
$$

Here, it is clear that we have not only the $u=0$ horizon, which in the new coordinates is the surface $t=x$, but another one at $t=-x$. In the old coordinates this is the surface $v=0$.

The basic property of event horizons, is the fact that they isolate certain space-time regions from another ones [8]. This can be demonstrated for the surfaces $t= \pm x$, in the following way if we restrict our attention to the $(t, x)$ plane.

Let us start by calling sector $I$, the region in the past of the horizons $(0<t< \pm x,-\infty<x<\infty)$. We also introduce the sector II, as the region to the future of the horizons $( \pm x<t<\infty,-\infty<x<\infty)$. From Eq. (23) we can see that null rays describe $45^{\circ}$ or $135^{\circ}$ straight lines in the $(t, x)$ plane. Therefore, not even the light will be able to return from sector II to sector $I$, once it has entered it. Although $t$ does not change the role of time with $x$ after crossing the horizons, as we shall see few important facts take place there.

The scalar field Eq. (10), may be obtained for the space-times being studied, in the new set of coordinates.

$$
\varphi(t, x)=\frac{1}{2} \sqrt{\frac{2 n}{2 n+1}} \ln \left[\frac{x+\left(x^{2}-t^{2}\right)^{1 / 2}}{x-\left(x^{2}-t^{2}\right)^{1 / 2}}\right] .
$$

If one inspects the expression of $\varphi(t, x)$ Eq. (24), one notices that for $t=0$ it diverges. Therefore, also for the present space-times we may interpret $t=0$ as a big bang singularity.

In sector $I, \varphi(t, x)$ Eq. (24) is a real function. On the other hand, in sector II the scalar field is purely imaginary. It means that in sector II, the stress-energy Eq. (2) may be negative and the scalar field will be an example of the so-called 'exotic matter'.

Observing $R$ Eq. (22) we see that it vanishes in both horizons but has the same sign in sectors $I$ and $I I$.

Finally, If one were interested in studying quantum field theory in sector II, the resulting theory would be unitary. This is the case because there is no singularities there and, from Eq. (14), the space-times under investigation possess a global timelike Killing vector field [11].

\section{Conclusions}

In the present work we have studied an analytical solution to the Einstein's equations in 2+1-dimensions. The space-time was dynamical and had a line symmetry. The matter content was represented by a minimally coupled, massless, scalar field.

The Einstein's equations for this system were identical to another one already solved in the literature. We have used the known solution $(S)$, and studied it.

We have imposed certain conditions upon $S$ such that it could be interpreted as a physically acceptable solution. These conditions restricted the domains of few free parameters $\left(p_{i}\right)$ of $S$, and divided $S$ in three distinct types of space-times, depending on the values of the $p_{i}$ 's. The first one was flat space-time. Then, we had a big bang model with a negative curvature scalar and a real scalar field. The last case was a big bang model with event horizons where the curvature scalar vanishes and the scalar field changes from real to purely imaginary.

\section{Acknowledgements}

I am grateful to A. Wang for suggestive discussions in the course of this work. I would like also to thank 
I. D. Soares for helpful discussions and FAPEMIG for the invaluable financial support.

\section{References}

[1] For a introduction see: R. Jackiw, in Physics, Geometry, and Topology, edited by H. C. Lee (Plenum, New York, 1990), pp. 191 - 239.

[2] M. Bañados, C. Teitelboim, and J. Zanelli, Phys. Rev. Lett. 69, 1849 (1992); M. Bañados, M. Henneaux, C. Teitelboim, and J. Zanelli, Phys. Rev. D 48, 1506 (1993).

[3] R. B. Mann and S. F. Ross, Phys. Rev. D 47, 3319 (1993); V. Husain, ibid 50, R2361 (1994); 526860 (1995); Y. Peleg and A. R. Steif, ibid 51, R3992 (1995).

[4] For a complete list of recent references see: S. Carlip,
Quantum Gravity in 2+1 Dimensions, (Cambridge University Press, Cambridge, 1998).

[5] G. Oliveira-Neto, Phys. Rev. D 58, 107501 (1998).

[6] J. Louko and P. J. Ruback, Class. Quantum Grav. 8, 91 (1991).

[7] D. Tsoubelis and A. Wang, J. Math. Phys. 32, 1017 (1991).

[8] C. W. Misner, K. S. Thorne and J. A. Wheeler, Gravitation, (Freeman, New York, 1973).

[9] A. H. Taub, J. Math. Phys. 29, 690 (1988).

[10] M. S. Morris, K. S. Thorne and U. Yurtsever, Phys. Rev. Lett. 61, 1446 (1988); M. S. Morris and K. S. Thorne, Am. J. Phys. 56, 395 (1988).

[11] B. S. DeWitt, Phys. Rep. 19C, 295-357 (1975); N. D. Birrel and P. C. W. Davies, Quantum fields in curved space, (Cambridge University Press, Cambridge, 1982). 